\title{
The unusual properties of anisotropic systems of quasiparticles in superfluid ${ }^{4} \mathrm{He}$
}

\author{
I.N. Adamenko, K.E. Nemchenko, and V.A. Slipko \\ V.N. Karazin Kharkiv National University, 4 Svobody Sq., Kharkiv 61077, Ukraine \\ E-mail: vaslipko@mail.ru \\ A.F.G. Wyatt \\ School of Physics, University of Exeter, Exeter EX4 4QL, UK \\ E-mail: a.f.g.wyatt@ex.ac.uk
}

Received October 1, 2006

\begin{abstract}
We discuss a number of unusual phenomena, which have been discovered recently in anisotropic quasiparticle systems in superfluid ${ }^{4} \mathrm{He}$. These include the creation of high-energy phonons by a pulse of low-energy phonons, the suprathermal distribution of high-energy phonons in long phonon pulses, the mesa shape of the angular distribution of low-energy phonons, the creation of a «hot line» when two-phonon pulses cross. The thermodynamic properties of anisotropic quasiparticle systems of He II are derived for all degrees of anisotropy.
\end{abstract}

PACS: 67.40.Db Quantum statistical theory; ground state, elementary excitations; 67.40.Fd Dynamics of relaxation phenomena.

Keywords: superfluid ${ }^{4} \mathrm{He}$, quasiparticle anisotropic systems, phonon, roton.

\section{Introduction}

In superfluid helium, it is possible to create quasiparticle systems which are strongly anisotropic (see, for example, Refs. 1-3). A phonon pulse is a strongly anisotropic quasiparticle system and can be created by a short current pulse of duration $t_{p} \sim 10^{-7}-10^{-5} \mathrm{~s}$ in a heater which is immersed in the superfluid helium. If the bulk temperature is $\sim 50 \mathrm{mK}$, then the influence of any ambient excitations, on the pulse, can be neglected, and the quasiparticle pulse moves in a «quasiparticle vacuum» from the heater to the detector. The quasiparticle pulse has a net momentum along the direction normal to the heater which defines the anisotropy axis. In momentum space, the distribution of occupied states is strongly anisotropic and this contrasts with isotropic quasiparticle systems which have no net momentum and have no special direction in momentum space.

We shall discuss a number of properties of anisotropic quasiparticle systems. In quasiequilibrium they can be characterized by a temperature $T$ and a drift velocity $\mathbf{u}$. In isotropic systems $u=0$, and in weakly anisotropic quasiparticle systems the drift velocity $u$ is much smaller than the maximum drift velocity $u_{\max }$. These have been studied for many years, but the properties of strongly aniso- tropic quasiparticle systems, when the drift velocity $u$ is near the maximum drift velocity $u_{\max }$, have not been considered until now.

\section{Quasiparticle anisotropic systems of superfluid helium: experiment and theory}

Anisotropic pulses of quasiparticles in superfluid helium are a unique physical system which show interesting and unusual behavior. One of the most amazing phenomena is the creation of high-energy phonons ( $h$-phonons) with energy $\varepsilon_{h} \sim 10 \mathrm{~K}$ from a pulse of low-energy phonons $\varepsilon_{\ell} \sim 1 \mathrm{~K}$ ( $\ell$-phonons $)$.

When a single short current pulse is applied to the heater, two-phonon pulses are detected by the bolometer (see Fig. 1). The faster pulse is due to $\ell$-phonons, and the slower one is due to $h$-phonons. Several experiments, including quantum evaporation of ${ }^{4} \mathrm{He}$ atoms from the free surface of liquid helium [4], unambiguously prove that $h$-phonons are produced in the bulk of liquid helium and not in the heater. The heater injects mainly $\ell$-phonons with short high-power pulses, but with long low-power pulses, it is possible to create rotons. The theory that a pulse of cold phonons can produce hot phonons has been presented in Refs. 5, 6. 


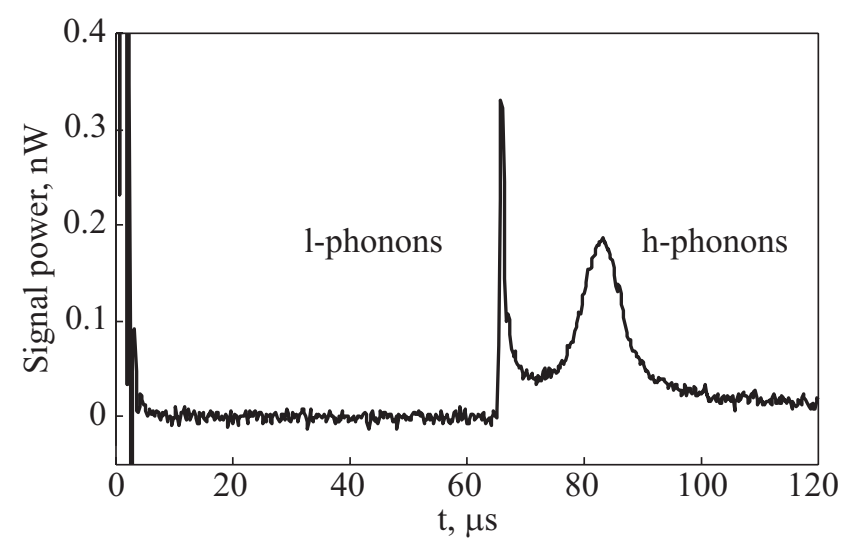

Fig. 1. The measured phonon signal in liquid ${ }^{4} \mathrm{He}$ showing the $\ell$ - and $h$-phonons produced by a single heater pulse: pulse duration $10^{-7} \mathrm{~s}$, heater power $10 \mathrm{~mW} / \mathrm{mm}^{2}$, propagation length $15.7 \mathrm{~mm}, T_{\mathrm{He}}=60 \mathrm{mK}$, at the saturated vapour pressure.

The $h$-phonons are created from the $\ell$-phonons as the system tries to reach equilibrium. The $\ell$-phonons in the pulse interact by four-phonon processes (4pp) and create pairs of low- and high-energy phonons. When the energy of the high-energy phonon is greater than $10 \mathrm{~K}$, it is very stable because it cannot spontaneously decay, unlike phonons with $\varepsilon<10 \mathrm{~K}$. The created $h$-phonons are lost from the back of the $\ell$-phonon pulse due to the difference in group velocities between the $\ell$ - and $h$-phonon subsystems $(238$ and $\leq 189 \mathrm{~m} / \mathrm{s}$, respectively), and create the second pulse. Whereas $h$-phonons interact weakly, $\ell$-phonon system attains quasiequilibrium very quickly by fast three-phonon processes (3pp). This causes the $\ell$-phonons to move as a whole with velocity which is close to a sound velocity $c=238 \mathrm{~m} / \mathrm{s}$. These phonons create the first pulse.

The theory accounts for the high efficiency of the $h$-phonon creation process, when up to $\sim 50$ per cent of the initial $\ell$-phonon energy along the anisotropy axis can be transformed to $h$-phonon energy. Using the exact drift distribution function, the energy density lost by the $\ell$ phonons, due to $h$-phonon creation, relative to the initial $\ell$-phonon density $\Delta(t)$, is shown as a function of time in Fig. 2.

Measurements of time-resolved angular distribution of $\ell$-phonons at several powers and pulse durations were made to investigate their temporal and spatial development [7]. Figure 3 shows angular distributions of $\ell$-phonon signal, integrated over time, for several input powers for $t_{p}=50 \mathrm{~ns}$. We can clearly see the constant central region with decreasing outer regions, in the shape of a mesa. The theory for the evolution of $\ell$-phonon pulses has been developed for the case of cool pulses [8], when $h$-phonon creation is neglected. In this case the evolution along and transverse to the anisotropy axis, are investi-

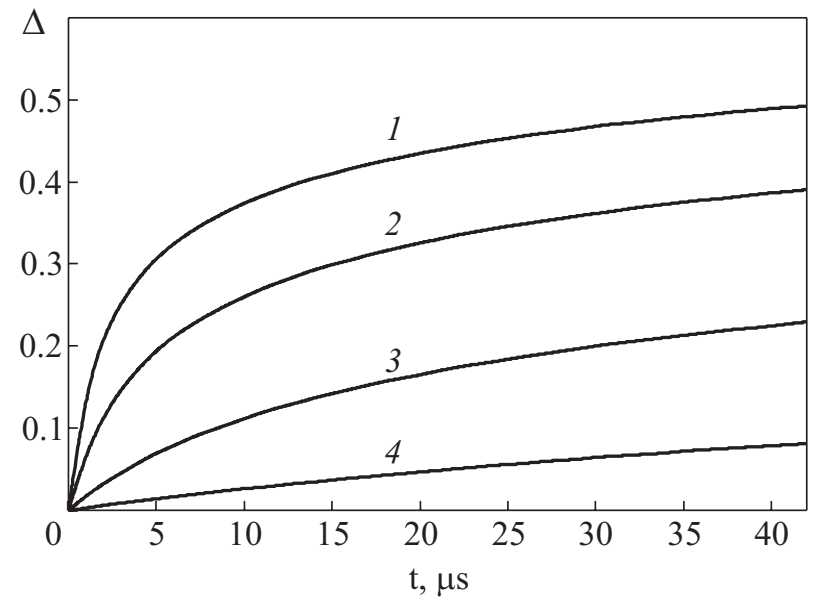

Fig. 2. The energy density lost by the $\ell$-phonons, due to $h$-phonon creation, relative to the initial $\ell$-phonon energy density $\Delta(t)$, as a function of time, calculated with $\chi=0.02$ for different values of $T$ equal to 0.041 (1), 0.036 (2), 0.030 (3), and 0.025 (4) K.

gated assuming instantaneous relaxation of the $\ell$-phonon system. This is a good approximation, because the characteristic time of three-phonon processes is less then any relevant time in the problem by two orders of magnitude. It was shown that in spite of the dispersion of $\ell$-phonon velocities, the $\ell$-phonon pulse moves as a whole with only a slow change in shape. This is in contrast to the case of noninteracting phonon pulse, which shows considerable dispersion.

The theory for the transverse evolution of long pulse gives the angular distribution for $\ell$-phonons due to lateral spreading. As it has a round shape, we suggest that mesa shape arises from $h$-phonon creation, the hotter central region of $\ell$-phonon pulse burns more quickly, whereas the

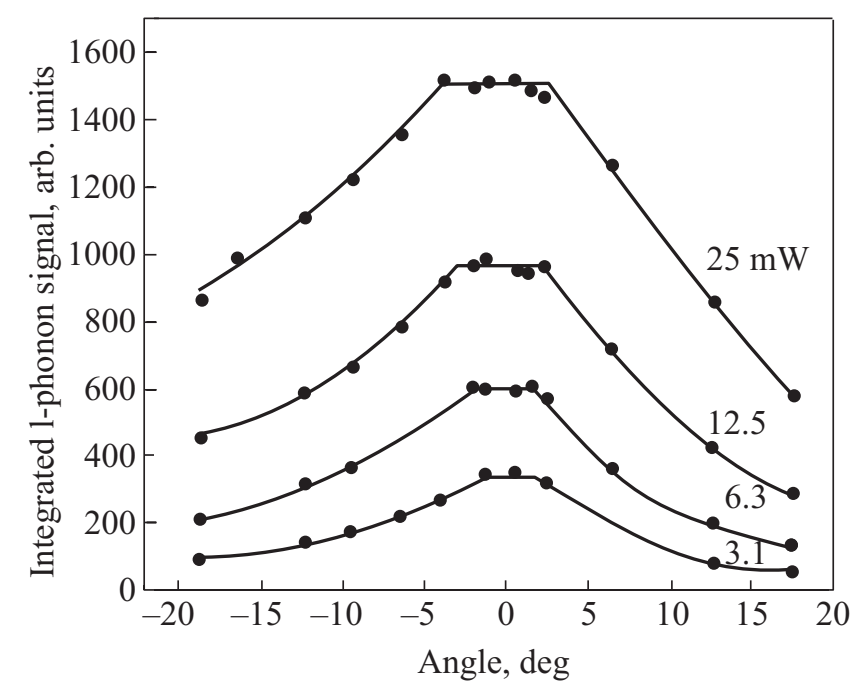

Fig. 3. The low-energy phonon signal, integrated over time, versus heater angle for a range of heater powers is shown. The pulse length is $50 \mathrm{~ns}$, propagation time is $16.7 \mathrm{~mm}$, and the lines are guides for the eye. 
cooler outer wings of $\ell$-phonon distribution are little changed. This is due to the very strong dependence of the $h$-phonon creation rate on $\ell$-phonon energy density. This interpretation is supported by the fact that the sum of $\ell$ and $h$-phonon angular distributions does not have a mesa shape but is rounded. It should be noted that the whole problem of the spatial evolution of the finite $\ell$-phonon pulse, with $h$-phonon creation taken consistently into account, is not yet theoretically solved.

Experiments have been performed in which two short phonon pulses (phonon sheets) are forced to collide [9]. When the angle between the normals to the two sheets is less then $13^{\circ}$, there is a strong interaction along the line of intersection of the two sheets. The amplitude of the $\ell$-phonon signal is greater than the sum of the signals from pulses which move independently to the bolometer (see Fig. 4). The interaction between the two sheets gets weaker as the angle between the two normals is larger then $13^{\circ}$. Also it has been found that the interaction is negligible when the pressure is increased to 19 bar.

The theory of this phenomenon has been developed [10]. If the time for the phonon pulses to cross the volume of overlap of the two sheet is greater than the time for phonon scattering $\tau_{3 p p}$, then phonons from the two pulses have enough time to interact with each other. The angle between the momenta of the two incoming phonons must be small for three-phonon processes to occur. This interaction creates a new anisotropic system, the hot line, which propagates along the total symmetry axis. The signals from the hot line are considerably greater than the sum of the signals from pulses which move independently to the bolometer (see Fig. 4) because energy is redirected,

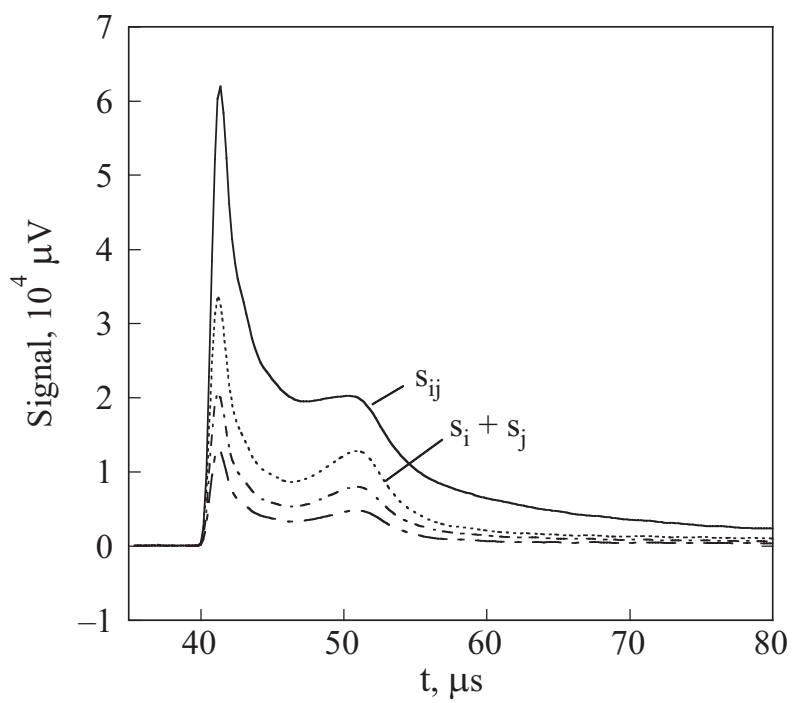

Fig. 4. The bolometer signals for the two separate pulses (dash-dotted lines) and for the double pulse (solid line). The sharp peak at $41 \mu$ s is due to the $\ell$-phonons; the signal after $\approx$ $44 \mu \mathrm{s}$ is due to $h$-phonons. The sum of the signals from the two separate pulses is shown as the dotted line. The input pulses are $100 \mathrm{~ns}$ and $6.3 \mathrm{~mW}$. from the shrinking phonon sheets, onto the bolometer in the form of the hot line.

If the time for the phonon pulses to cross the volume of overlap of the two sheets is less than the time for phonon scattering $\tau_{3 p p}$, then the phonons from the two pulses have no time to interact. This occurs when the angle between two sheets is large or when the $3 p p$ scattering rate is reduced by applying pressure. Then the hot line does not form because the pulses pass through each other without interaction.

In addition to the «dynamical phenomena» considered above, there are very interesting «static properties», in particular, thermodynamic properties of anisotropic quasiparticle systems, which we shall discuss in the following sections.

\section{Quasiequilibrium distribution functions of anisotropic quasiparticle systems}

For isotropic quasiparticle systems there is no special direction as all directions are equivalent. In this case the distribution function depends on the modulus of momentum $p$, and the momentum density of quasiparticles is zero.

Anisotropic quasiparticle systems have a special direction in momentum space, and the distribution function $n$ depends on the vector of momentum $\mathbf{p}$, and the momentum density $\mathbf{P}$,

$$
\mathbf{P}=\int_{\Gamma_{p}} \mathbf{p} n(\mathbf{p}) \frac{d^{3} p}{(2 \pi \hbar)^{3}},
$$

is not zero (here $\Gamma_{p}$ is the region of integration in momentum space).

The equilibrium distribution function of anisotropic quasiparticle systems must make the collision integral equal to zero. Also the momentum density should be nonzero. These conditions are satisfied by the Bose-Einstein distribution which is a function of the energy $\varepsilon=\varepsilon(p)$ of quasiparticles, the temperature $T$ and the drift velocity $\mathbf{u}$ :

$$
n(\mathbf{p})=\left[\exp \left(\frac{\varepsilon(p)-\mathbf{p} \cdot \mathbf{u}}{k_{B} T}\right)-1\right]^{-1} .
$$

The distribution function $n$ must be positive. As a result, the drift velocity $u$ can change up to the minimum value of the phase velocity of the quasiparticles, which form the system:

$$
0 \leq u \leq u_{\max }, \text { where } u_{\max }=\left(\frac{\varepsilon(p)}{p}\right)_{\min },
$$

which is determined by the dependence of the quasiparticle energy $\varepsilon$ on its momentum $p$. 
The energy-momentum relation for superfluid helium is well known (see, for example, [11]). It contains a phonon part $\varepsilon_{\mathrm{ph}}(p)$, which has a nearly linear dependence and the roton part $\varepsilon_{r}(p)$, which can be described by a parabola:

$$
\varepsilon_{\mathrm{ph}}(p)=c p(1+\psi(p)), \text { and } \varepsilon_{r}(p)=\Delta+\frac{\left(p-p_{0}\right)^{2}}{2 \mu},
$$

where the parameters for the roton spectrum are $\Delta / k_{B}=8.71 \mathrm{~K}, p_{0} / \hbar=1.91 \AA^{-1}, \quad \mu=0.161 m_{4}$, and $m_{4}$ is the ${ }^{4} \mathrm{He}$ atomic mass, at zero pressure.

The function $\psi(p)$, which describes the deviation from linearity for phonon energy-momentum relation, is small $(|\psi(p)|<<1)$. Nevertheless it completely determines the type and strength of phonon interactions. According to [12], at low pressure, the function $\psi(p)$ is positive for phonons with momentum $0<p<p_{c}$. This is the momentum range of the $\ell$-phonons where the fastest scattering is 3 pp. For small $p$ the function $\psi(p) \sim p^{2}$. At $c p / k_{B} \approx 7 \mathrm{~K}$ the function $\psi(p)$ reaches its maximum value $\approx 0.04$. After that the function $\psi(p)$ decreases and becomes zero at $p=p_{c}$. For $p>p_{c}, \psi(p)$ is negative, so $3 p p$ is prohibited by the conservation laws. In this case the fastest scattering is $4 \mathrm{pp}$. This is the momentum condition for $h$-phonons. At zero pressure $c p_{c} / k_{B}=10 \mathrm{~K}$. We shall use for numerical calculations a convenient analytical approximation of the function $\psi(p)$ introduced in Ref. 13 .

It follows from Eqs. (3) and (4) for the pure phonon system, that the minimum phase velocity $u_{\mathrm{ph}}$ is close to the sound velocity $c$. For the phonon-roton system, the minimum phase velocity $u_{r} \approx \Delta / p_{0}$ is determined by the roton minimum.

Besides condition (3), the drift velocity $u$ must have a value which makes the system thermodynamically stable. The general thermodynamic inequality [14] that can be applied to superfluid helium, determines the line of thermodynamic stability $u=u_{\text {st }}(T)$. We have derived (see Refs. 13, 15) the stability curves for anisotropic phonon and phonon-roton systems. The results are presented on Fig. 5. At $T=0$ the respective maximum drift velocity coincides with the Landau critical velocity for the corresponding quasiparticle system. The critical velocity decreases monotonically with increasing temperature, but remains close to the $u_{\max }$ for temperatures up to $T \sim 1 \mathrm{~K}$. So, strongly anisotropic phonon (Fig. 5,a) and phononroton systems (Fig. 5,b) are thermodynamically stable up to high temperatures.

The quasiequilibrium state is realized in the pulse for phonons whose time of relaxation $\tau_{\mathrm{ph}}(\varepsilon)$ is smaller than the current pulse duration $t_{p}$. Our calculations show [6] that, in long sufficiently pulse $\left(t_{p}>10^{-7} \mathrm{~s}\right)$, there are phonons with energy up to $11 \mathrm{~K}$ in the quasiequilibrium state. So the quasiequilibrium phonon system can be char-
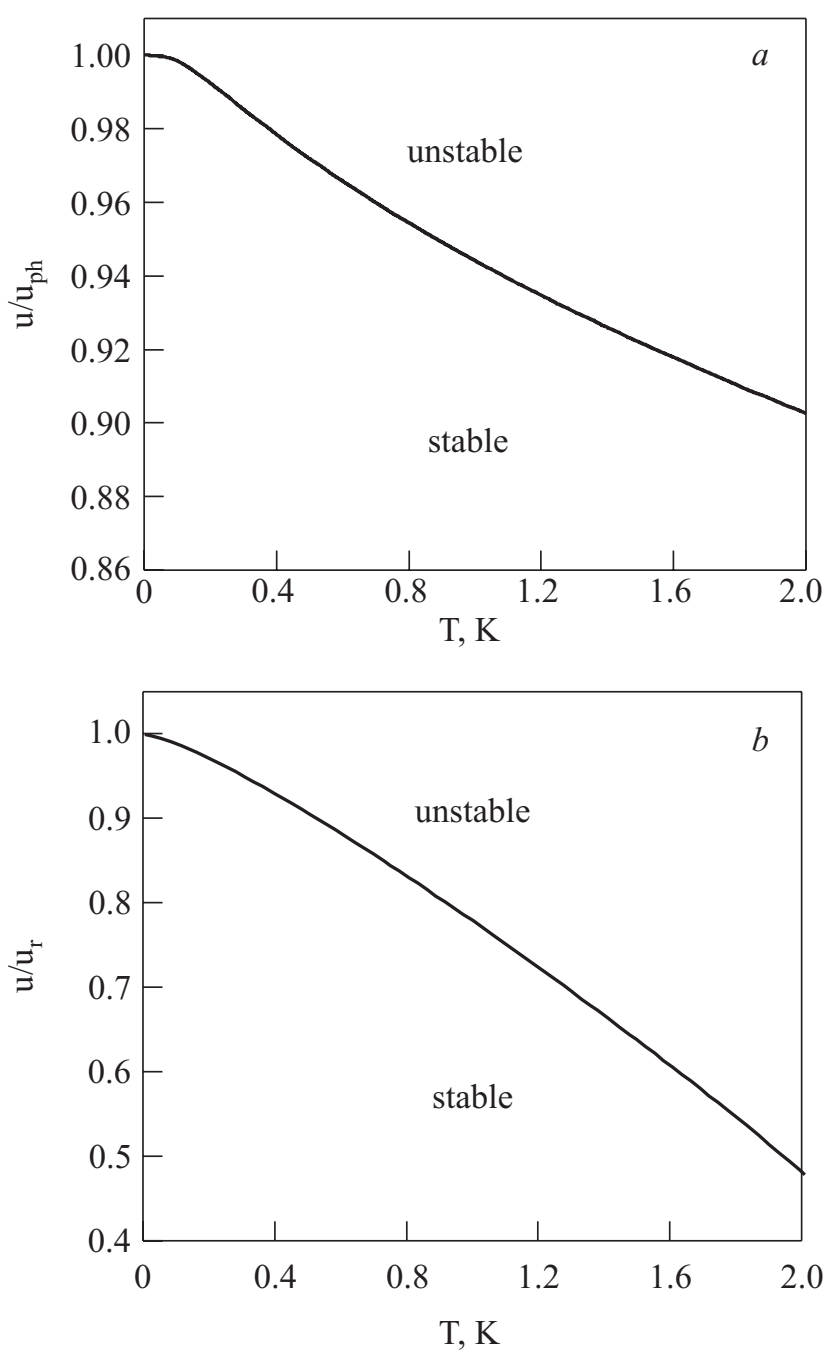

Fig. 5. The maximum thermodynamically stable drift velocity divided by $u_{\max }$ for anisotropic phonon $\left(p_{f}=11 \mathrm{~K}\right)(a)$ and for anisotropic phonon-roton $(b)$ as a function of temperature.

acterized by the Bose-Einstein distribution (2) up to some phonon momentum $p_{f} \leq 11 \mathrm{~K}$.

Let us introduce $\zeta=1-\cos \theta$, where the angle $\theta$ is between the phonon momentum $\mathbf{p}$ and the anisotropy axis $z$, directed along the vector of drift velocity $\mathbf{u}$. Also, we introduce the parameter of anisotropy $\chi=1-u / c$. Then the quasiequilibrium phonon distribution function $n_{\mathrm{ph}}$ can be rewritten in a form useful for analyzis:

$$
n_{\mathrm{ph}}(p, \zeta)=\left[\exp \left(\frac{c p}{k_{B} T}\{\chi+\psi(p)+\zeta(1-\chi)\}\right)-1\right]^{-1}
$$

For the isotropic phonon system the parameter of anisotropy $\chi$ is equal to unity, and the curly bracket, in the exponent of the expression (5), is close to unity. For the strongly anisotropic phonon systems, the anisotropy parameter $\chi$ is much less than unity. $\psi(p)$ is also small and the curly bracket, in the exponent is small, when $\zeta$ is small. As a result, when the angle $\theta$ is small we have a 


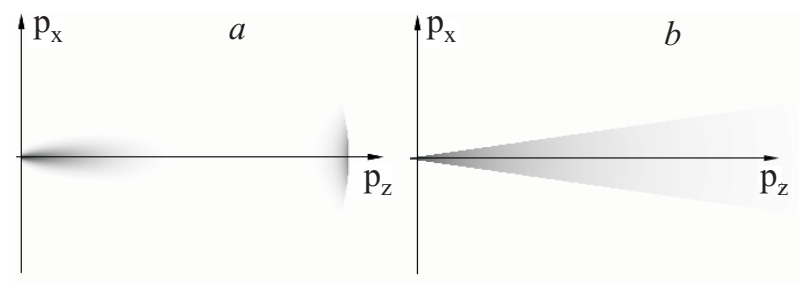

Fig. 6. The exact distribution function $(a)$ is shown schematically for typical values in experiments $\chi=0.04, T=0.05 \mathrm{~K}$ and $p_{f}=11 \mathrm{~K}$ along with the conus distribution function $(b)$ with cone angle $\theta_{c}=12^{\circ}$ and the temperature $T_{p}=1 \mathrm{~K}$.

large number of phonons. This situation in momentum space, is shown schematically on Fig. 6, $a$ for typical values in experiments $\chi=0.04$ and $T=0.05 \mathrm{~K} ; p_{f}=11 \mathrm{~K}$.

In directions close to the anisotropy axis, there are a large number of high-energy phonons with energy higher than $10 \mathrm{~K}$, because for these phonons $\psi(p)$ is negative and cancels $\chi$ in the distribution function (5). As a result, the curly bracket, in the exponent, is smallest for $h$-phonons. The large number of high-energy phonons in a longphonon pulse we call a suprathermal distribution (see Refs. 16, 17).

In the direction perpendicular to the $z$ axis there are the same number of phonons as in the isotropic case at $T=0.05 \mathrm{~K}$. This is because, for the perpendicular direction, $\zeta$ is equal to unity and the curly bracket, in the distribution function (5), is also close to unity. At this temperature, the number of phonons is very small. So we can have the unusual situation in a strongly anisotropic phonon system; the temperature of the pulse can be lower than the temperature of superfluid helium into which it is injected, but the pulse has a much larger number of phonons than the helium.

We can define a cone in momentum space, which is cut from the isotropic Bose distribution for phonons (see Fig. 6,b):

$$
n_{c}(p, \theta)=\frac{\eta\left(\theta_{c}-\theta\right)}{\mathrm{e}^{c p / k_{B} T_{p}}-1} .
$$

If this cone has a cone angle $\theta_{c}$ of $12^{\circ}$ and the temperature $T_{p}$ of the isotropic distribution is $1 \mathrm{~K}$, then the energy density and momentum density, is equal to the anisotropic system defined by the typical values of $\chi=0.04$ and $T=0.05 \mathrm{~K}$, which were mentioned above. The distribution function $n_{c}$, which includes the step function $\eta$, is the Bose-cone approximation. Note that in this cone approximation, the suprathermal distribution is absent.

\section{Energy and angular distributions of anisotropic quasiparticle systems}

Figure 7,a shows the momentum dependence for the anisotropic phonon system with $T=0.05 \mathrm{~K}$ and $\chi=0.04$ of the energy distribution function $E(p)$, which is determined for all quasiequilibrium quasiparticle systems by the following expression:

$E(p)=\int_{0}^{2} \varepsilon n(p, \zeta) p^{2} d \zeta=-\frac{k_{B} T p \varepsilon}{u}\left\{\ln \left[1-\exp \left(-\frac{\varepsilon(p)-p u}{k_{B} T}\right)\right]-\right.$

$\left.-\ln \left[1-\exp \left(-\frac{\varepsilon(p)+p u}{k_{B} T}\right)\right]\right\}$.

The energy distribution for the Bose function, when the drift velocity is equal to zero, has one maximum, as is
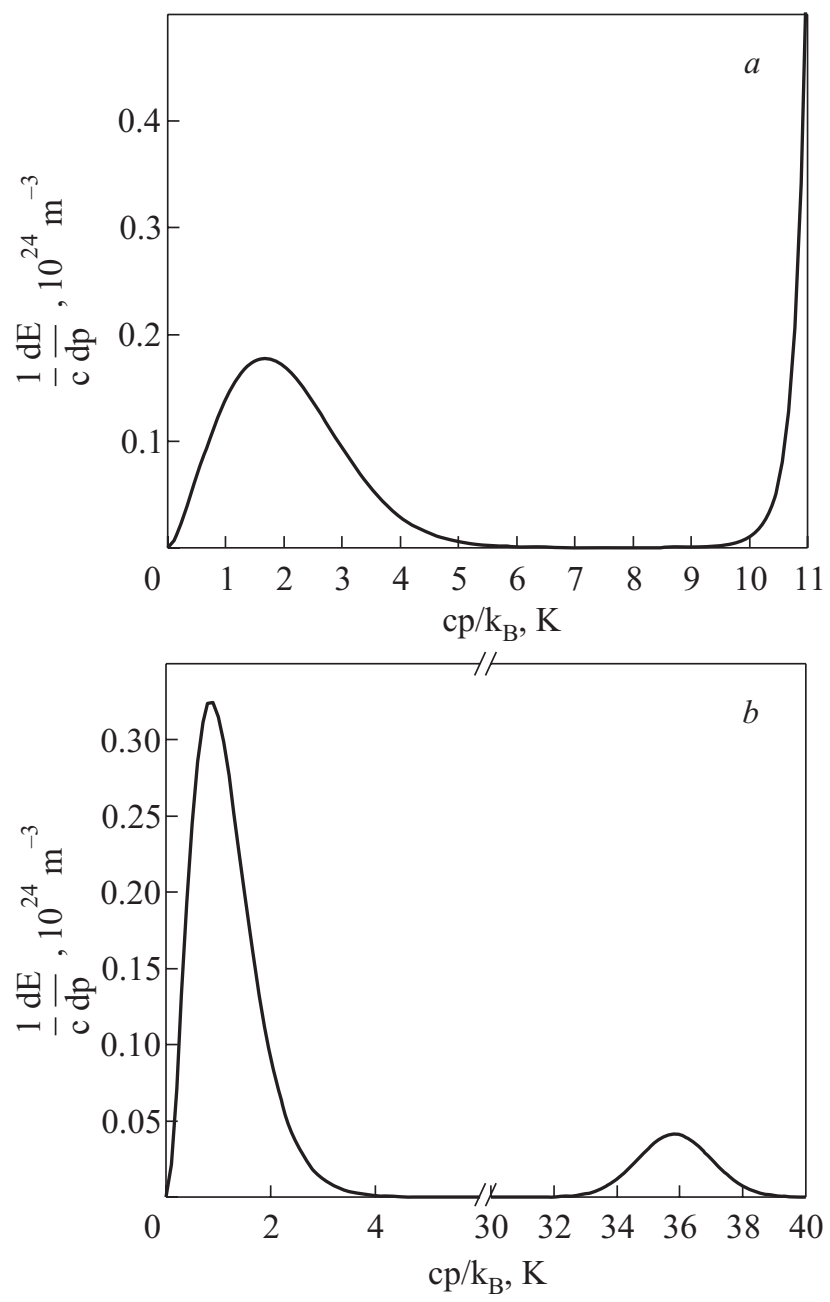

Fig. 7. The momentum dependence of the quasiparticle energy distribution for the anisotropic phonon system with $T=0.05 \mathrm{~K}$, $\chi=0.04(a)$ and for the anisotropic phonon-roton system with $T=0.3 \mathrm{~K}, u / u_{r}=0.63(b)$, which corresponds the same energy and momentum density as for Fig. 7,a. 
well known. For a strongly anisotropic phonon system, with typical experimental values $\chi$ and $T$, we see there are two almost separate subsystems: a subsystem of low-energy phonons, which forms the first maximum and a subsystem of high-energy phonons, which forms the second maximum. The second maximum is caused by the negative value of dispersion function $\psi(p)$ for $h$-phonons, which cancels $\chi$ in Eq. (5), and is the suprathermal distribution.

In Fig. 7, $b$ we see the momentum dependence of the energy distribution function $E(p)$ for the anisotropic phonon-roton system, which has the same energy and momentum density as the phonon system in Fig. 7,a, but now $T=0.3 \mathrm{~K}$ and $u / u_{r}=0.63$. Even though the temperature is low, the roton energy density is very high compared to that in the isotropic phonon-roton system with $u=0$ and $T=0.3 \mathrm{~K}$. Anisotropic phonon-roton systems are realized when we have superfluid flow in narrow channels where the normal component is at rest. Also they should be formed in very long pulses in superfluid helium.

It is natural to define the normalized angular distribution function, which characterizes the anisotropy of a quasiparticle subsystem, as follows:

$$
W_{p_{1}-p_{2}}(\zeta)=\frac{\int_{p_{1}}^{p_{2}} \varepsilon n(p, \zeta) p^{2} d p}{\int_{0}^{2} d \zeta \int_{p_{1}}^{p_{2}} \varepsilon n(p, \zeta) p^{2} d p} .
$$

Figure $8, a$ shows angular distribution function $W_{p_{1}-p_{2}}(\zeta)$ for the $\ell$ - and $h$-phonons, that form the first and second maxima in Fig. 7, $a$. Note, that the $h$-phonons are concentrated near the anisotropy axis, with a narrower angular distribution than $\ell$-phonons. This behavior was observed in [18].

In Fig. $8, b$ we see that phonons form a weakly anisotropic background, while the roton subsystem is strongly anisotropic. It is interesting to note that most of the energy is in the phonon subsystem, whereas the momentum is mainly in the roton subsystem.

\section{Thermodynamic functions of anisotropic quasiparticle systems}

The thermodynamic functions of the gas of quasiparticles can be found from the expression for the free energy density $F$ :

$$
F=k_{B} T \int \ln \left[1-\exp \left(-\frac{\varepsilon(p)-\mathbf{p} \cdot \mathbf{u}}{k_{B} T}\right)\right] \frac{d^{3} p}{(2 \pi \hbar)^{3}},
$$

which we obtain for all levels of anisotropy,
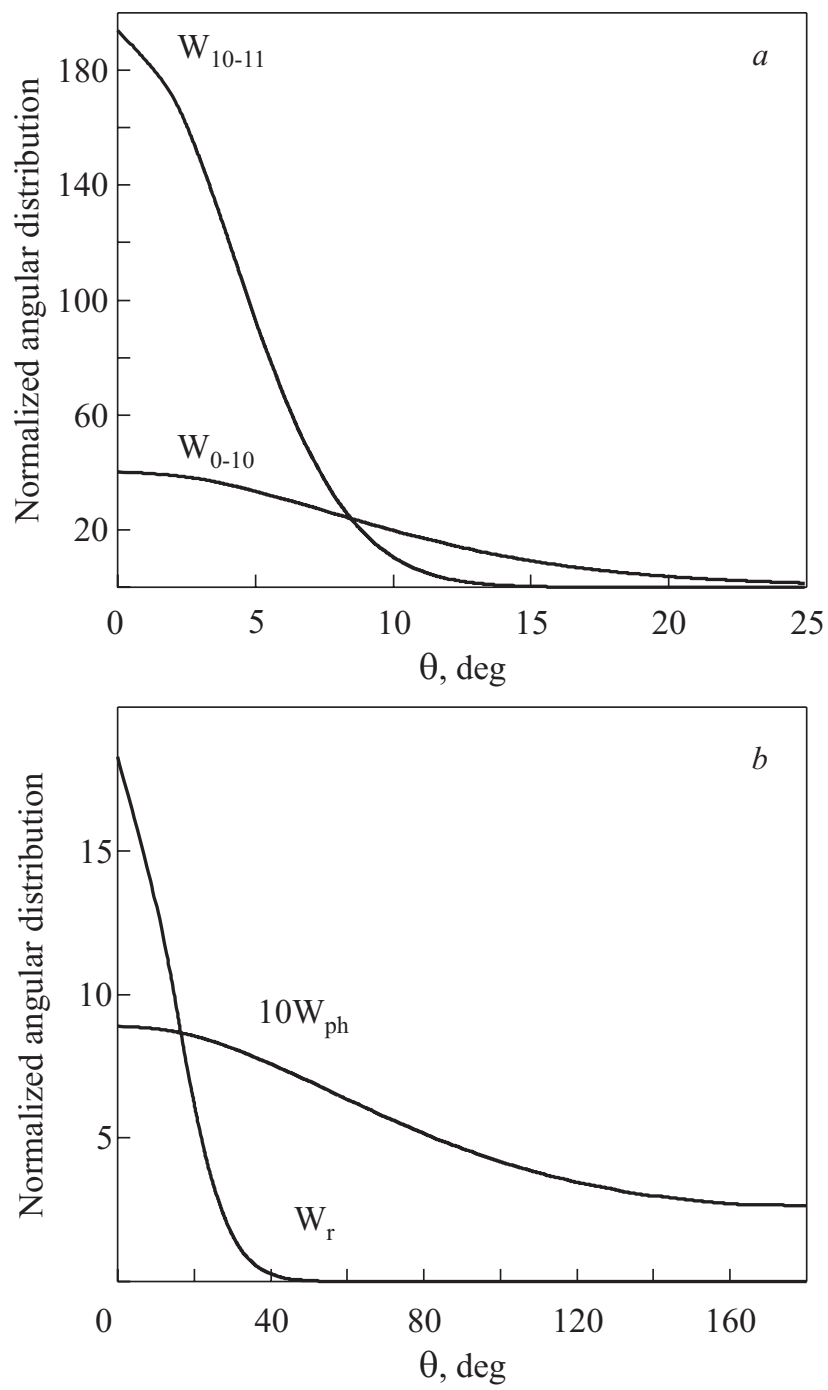

Fig. 8. $a-$ Angular distribution function $W_{p_{1}-p_{2}}(\theta)$ for the $\ell$ and $h$-phonons, that form the first and second maxima in Fig. 7, $a . b-$ Angular distribution function $W_{p_{1}-p_{2}}(\theta)$ for phonon and roton subsystems, that form the first and second maxima in Fig. 7,b.

$F=-\frac{\left(k_{B} T\right)^{2}}{4 \pi^{2} \hbar^{3} u} \int p d p\left\{L_{2}\left(\frac{\varepsilon(p)-p u}{k_{B} T}\right)-L_{2}\left(\frac{\varepsilon(p)+p u}{k_{B} T}\right)\right\}$,

where

$$
L_{\alpha}(x)=\sum_{n=1}^{\infty} \frac{1}{n^{\alpha}} \mathrm{e}^{-x n}
$$

is a polylogarithmic function of $\alpha$ th order.

At low values of $x$, which applies, for example, to a strongly anisotropic roton gas, it is useful to rewrite the series (11) as a series in powers of $x$ : 


$$
\begin{aligned}
& L_{\alpha}(x)=\Gamma(1-\alpha) x^{\alpha-1}+ \\
& +\sum_{n=0}^{\infty} \frac{(-1)^{n+1} \Gamma(1+n-\alpha)}{\pi n !(2 \pi)^{n-\alpha}} \sin \left[\frac{\pi}{2}(n-\alpha)\right] \zeta(1+n-\alpha) x^{n},
\end{aligned}
$$

where $\Gamma$ is Euler gamma-function; $\zeta$ is Riemann zeta-function.

The entropy density $S$, heat capacity density $C$, the momentum density $\mathbf{P}$, and the density of the normal component $\rho_{n}$ can be obtained by differentiating the free energy density $F$ :

$$
S=-\frac{\partial F}{\partial T}, C=-T \frac{\partial^{2} F}{\partial T^{2}}, \quad \mathbf{P}=-\frac{\partial F}{\partial \mathbf{u}}, \rho_{n}=\frac{P}{u} .
$$

For differentiation, one should note that $d L_{\alpha}(x) / d x=$ $=-L_{\alpha-1}(x)$.

Our calculation shows that the temperature dependence of the thermodynamic functions for a strongly anisotropic phonon system, is essentially different to the isotropic case. The values of the thermodynamic functions, of strongly anisotropic phonon systems, are much larger than for the isotropic system at the same temperature, because they occupy different volumes in momentum space. So, for example, at the same low temperature, the energy density of a strongly anisotropic phonon system with typical value $\chi$ is more than three orders larger than the isotropic one. This fact is also illustrated by Fig. 9, where the temperature dependence of entropy density $S_{\mathrm{ph}}\left(u=u_{\mathrm{st}}\right)$ of the extremely anisotropic phonon system divided by entropy density of isotropic phonon system $(u=0)$, is shown.

The general expression (10) can be simplified for the roton system:

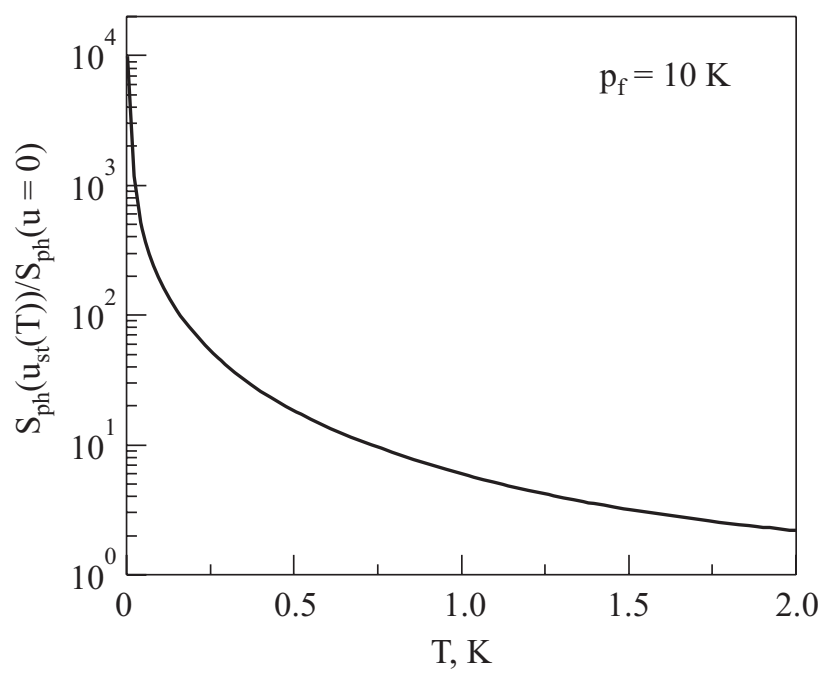

Fig. 9. The temperature dependence of entropy density $S_{\mathrm{ph}}\left(u=u_{\mathrm{st}}\right)$ of the extremely anisotropic phonon system divided by entropy density of the isotropic phonon system $(u=0)$.

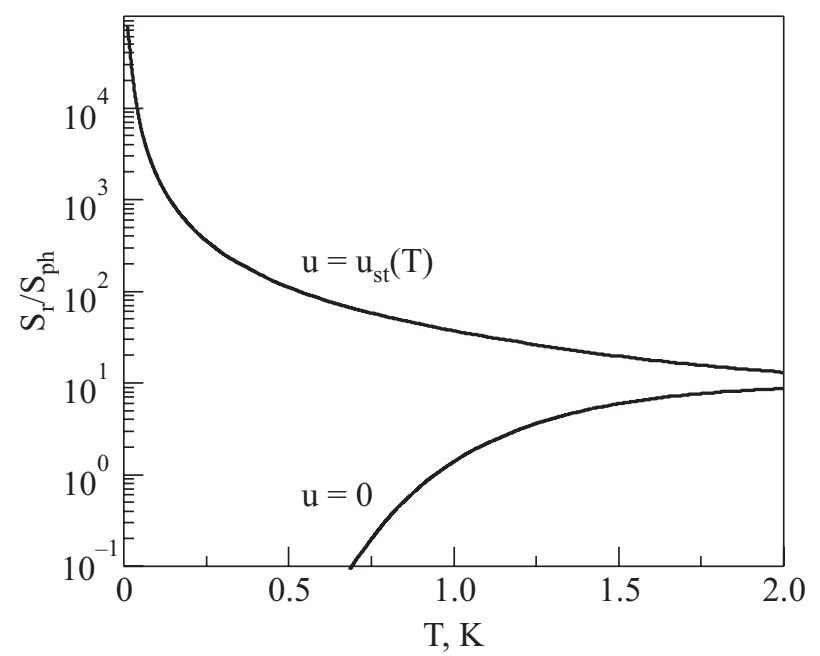

Fig. 10. The temperature dependence of the roton entropy density $S_{r}$ divided by the phonon entropy density $S_{\mathrm{ph}}$ on the line of thermodynamic stability and for the isotropic, $u=0$, system.

$F_{r}=-\frac{\sqrt{\mu} p_{0}\left(k_{B} T\right)^{5 / 2}}{(2 \pi)^{3 / 2} \hbar^{3} u}\left\{L_{5 / 2}\left(\frac{\Delta^{*}(u)}{k_{B} T}\right)-L_{5 / 2}\left(\frac{\Delta^{*}(-u)}{k_{B} T}\right)\right\}$,

where we denote $\Delta^{*}(u)=\Delta-p_{0} u-\mu u^{2} / 2$.

It should be noted, that the third term in $\Delta^{*}(u)$ cannot be omitted, because in highly anisotropic systems (at $u \approx u_{\max }$ ) it can have a similar value to the difference of the first and second terms.

If $u=u_{\max }$, then $\Delta^{*}(u)=0$, and the function $L_{5 / 2}(0)$ is equal to $\zeta(5 / 2)$. In this case, when the temperature decreases, the free energy of the strongly anisotropic roton subsystem, decreases as $T^{5 / 2}$, according to Eq. (14). At the same time the free energy of the weakly anisotropic phonon system, varies as $T^{4}$ [11].

This temperature dependence gives an unusual property: in strongly anisotropic phonon-roton system of superfluid helium, all the thermodynamic properties of helium, at all temperatures, are determined by rotons. The dominance of the rotons over the phonons can also be seen in the Fig. 10, where the temperature dependence of the roton entropy density $S_{r}$ divided by the phonon entropy density $S_{\text {ph }}$ are presented on the line of thermodynamic stability, and also for the isotropic systems where $u=0$. This behavior is in strong contrast to the isotropic quasiparticle system, where rotons «freeze out» faster than phonons, and so for temperatures less then $0.5 \mathrm{~K}$ the roton contribution can be neglected.

\section{Conclusion}

We see that the strongly anisotropic phonon and phonon-roton systems are thermodynamically stable over a wide temperature range and can be realized in experi- 
ments. A number of unusual phenomena have been discovered recently such as the phenomenon of the creation of high-energy phonons by a pulse of low-energy phonons; the suprathermal distribution of high-energy phonons in long phonon pulses; the mesa shape angular distribution of low-energy phonons; the phenomenon of the «hot line» formation, when two phonon pulses cross.

The theoretical results on anisotropic quasiparticle systems considered in this paper are:

1. The energy distribution of a strongly anisotropic phonon system has two maxima. The second maximum, is formed by $h$-phonons, and it demonstrates a suprathermal distribution, which is realized in long pulses.

2 . The $h$-phonons are concentrated near the anisotropy axis; they have smaller angular distribution than $\ell$-phonons, which was observed in Ref. 18.

3 . The values of the thermodynamic functions, of strongly anisotropic phonon systems, are much larger than for isotropic systems at the same temperature.

4. In the strongly anisotropic phonon-roton system, all thermodynamic properties of helium, at all temperatures, are determined by rotons.

5. The thermodynamic functions of strongly anisotropic phonon and phonon-roton systems have unusual temperature dependencies, which are essentially different from the isotropic system.

Although a number of astonishing facts have already been discovered, we think that the physics of anisotropic quasiparticle systems will show more surprising phenomena in the future.

We express our gratitude to EPSRC, UK (grant EP/C $523199 / 1)$ for supporting this work.
1. A.F.G. Wyatt, N.A. Lockerbie, and R.A. Sherlock, J. Phys.: Condens. Matter 1, 3507 (1989).

2. A.F.G. Wyatt and M. Brown, Physica B165-166, 495 (1990).

3. M.A.H. Tucker and A.F.G. Wyatt, J. Phys.: Condens. Matter 6, 2813 (1994).

4. M.J. Baird, F.R. Hope, and A.F.G. Wyatt, Nature 304, 325 (1983).

5. I.N. Adamenko, K.E. Nemchenko, A.V. Zhukov, M.A.H. Tucker, and A.F.G. Wyatt, Phys. Rev. Lett. 82, 1482 (1999).

6. I.N. Adamenko, Yu.A. Kitsenko, K.E. Nemchenko, V.A. Slipko, and A.F.G. Wyatt, Phys. Rev. B73, 134505 (2006).

7. R. Vovk, C.D.H. Williams, and A.F.G. Wyatt, Phys. Rev. B68, 134508 (2003).

8. I.N. Adamenko, K.E. Nemchenko, V.A. Slipko, and A.F.G. Wyatt, Phys. Rev. B68, 134507 (2003).

9. R. Vovk, C.D.H. Williams, and A.F.G. Wyatt, Phys. Rev. Lett. 91, 235302 (2003).

10. I.N. Adamenko, Yu.A. Kitsenko, K.E. Nemchenko, V.A. Slipko, and A.F.G. Wyatt, Phys. Rev. B72, 054507 (2005).

11. I.M. Khalatnikov, An Introduction to the Theory of Superfluidity, W.A. Benjamin (ed.), New York-Amsterdam (1965).

12. W.G. Stirling, in: 75th Jubilee Conference on Liquid Helium-4, J.G.M. Armitage (ed.), World Scientic, Singapore (1983), p. 109; and private communication.

13. I.N. Adamenko, K.E. Nemchenko, V.A. Slipko, and A.F.G. Wyatt, J. Phys.: Condens. Matter 18, 2805 (2006).

14. A.F. Andreev and L.A. Melnikovsky, JETP Lett. 78, 574 (2003) [Pis'ma v JETF 78, 1063 (2003)].

15. I.N. Adamenko, K.E. Nemchenko, V.A. Slipko, and A.F.G. Wyatt, Phys. Rev. Lett. 96, 065301 (2006).

16. A.F.G. Wyatt, M.A.H. Tucker, I.N. Adamenko, K.E. Nemchenko, and A.V. Zhukov, Physica B280, 36 (2000).

17. I.N. Adamenko, K.E. Nemchenko, and A.F.G. Wyatt, Low Temp. Phys. 29, 11 (2003) [Fiz. Nizk. Temp. 29, 16 (2003)].

18. M.A.H. Tucker and A.F.G. Wyatt, Physica B194, 551 (1994). 